\title{
O impacto do coronavírus na doença de Alzheimer: uma revisão narrativa
}

The impact of coronavirus on Alzheimer's disease: a narrative review

El impacto del coronavirus en la enfermedad de Alzheimer: una revisión narrativa

Julio Cesar Pinheiro da Rocha Junior ${ }^{1 *}$, Gabriela de Oliveira Melo, Veronica Nunes da Silva Cardoso $^{2}$, Graziela Santos da França ${ }^{1}$, Gleydiane de Oliveira Silva ${ }^{1}$, Vivian Nogueira Gentil².

\section{RESUMO}

Objetivo: Analisar através de uma revisão narrativa o impacto do isolamento social diante da pandemia COVID-19 para o cliente idoso com doença de Alzheimer e sua família. Revisão bibliográfica: Os pacientes com Doença de Alzheimer (DA) são vulneráveis a desastres e crises, por causa de suas deficiências neurocognitivas e rica sintomatologia neuropsiquiátrica. Isso é especialmente verdadeiro durante a pandemia do COVID-19, visto que os idosos com DA perderam o contato com familiares e amigos, deixando de realizar suas atividades, ficando ainda mais isolados e apresentando maiores alterações neuropsiquiátricas como depressão, ansiedade, agitação, apatia, atividade motora aberrante e estresse, assim como a família ficou mais sobrecarregada. Considerações finais: Diante do exposto foi possível analisar o impacto do coronavírus no paciente idoso com Alzheimer e sua família, constatando que discutir sobre essa temática é de extrema importância, pois em virtude da pandemia, os idosos com DA ficaram ainda mais vulneráveis e a família ficou muito mais sobrecarregada e abalada.

Palavras-chave: Infecções por Coronavírus, Doença de Alzheimer, Idoso.

\begin{abstract}
Objective: To analyze through a narrative review the impact of social isolation in the face of the COVID-19 pandemic for the elderly client with Alzheimer's disease and his family. Literature review: The elderly with Alzheimer's disease (AD) are vulnerable to disasters and crises due to their neurocognitive deficits and many neuropsychiatric symptoms. This is more evident during the COVID-19 pandemic, as elderly people with AD lost contact with family and friends, stopping their activities, becoming even more isolated and showing greater neuropsychiatric changes such as depression, anxiety, agitation, apathy, aberrant motor activity and stress, just as the family became more overwhelmed. Final considerations: Given the above, it was possible to analyze the impact of the coronavirus on the elderly patient with Alzheimer's and his family, noting that discussing this theme is extremely important, since due to the pandemic, the elderly with AD became even more vulnerable and the family was much more overwhelmed and shaken.
\end{abstract}

Key words: Coronavírus infections, Alzheimer disease, Aged.

\section{RESUMÉN}

Objetivo: Analizar, a través de una revisión narrativa, el impacto del aislamiento social frente a la pandemia de COVID-19 en pacientes ancianos con Alzheimer y sus familias. Revisión de la literatura: los pacientes con enfermedad de Alzheimer (EA) son vulnerables a desastres y crisis debido a sus deficiencias neurocognitivas y su rica sintomatología neuropsiquiátrica. Esto es especialmente cierto durante la pandemia de COVID-19, ya que las personas mayores con EA perdieron el contacto con familiares y amigos, dejando de realizar sus actividades, volviéndose aún más aisladas y presentando mayores cambios neuropsiquiátricos como depresión, ansiedad, agitación, apatía, actividad motora aberrante y el estrés, así como las familias se volvieron más sobrecargadas. Consideraciones finales: Dado lo anterior, se pudo analizar el impacto del coronavirus en los pacientes ancianos con Alzheimer y sus familias, señalando que discutir este tema es sumamente importante, ya que, debido a la pandemia, las personas mayores con EA se volvieron aún más vulnerables y la familia se sintió mucho más sobrecarregada y conmocionada.

Palabras clave: Infecciones por Coronavirus, Enfermedad de Alzheimer, Anciano.

${ }^{1}$ Centro Universitário IBMR, Rio de Janeiro - RJ. *E-mail: jcprjß@gmail.com

2 Universidade Federal do Rio de Janeiro, Rio de Janeiro - RJ. 


\section{INTRODUÇÃO}

A Doença de Alzheimer (DA) é uma doença neurodegenerativa, progressiva e irreversível, que degenera progressivamente o nível de consciência do indivíduo, e posteriormente afeta o funcionamento de todo o corpo. Um levantamento realizado pela Organização Mundial da Saúde (OMS) evidenciou que, até 2050, em média 152 milhões de pessoas irão receber o diagnóstico de demência, representando $70 \%$ dos casos. Segundo dados do Sírio Libanês, em 2018 o mundo apresentava 35,6 milhões de casos da DA e, no Brasil, o valor chegou a 1,2 milhão (SÍRIO-LIBANÊS, 2018).

A DA está entre as principais causas de dependência e deficiência entre os idosos no mundo inteiro. Estima-se que, em todo o mundo, cerca de 50 milhões de pessoas possuem DA, com quase 10 milhões de casos novos todo ano. Em 2030, o número total de pessoas com DA está projetado para chegar em 82 milhões e em 2050 em 152 milhões de pessoas (PRINCE M, et al., 2016).

Por conseguinte, em virtude de suas deficiências cognitivas e funcionais, os pacientes com DA são mais vulneráveis em períodos de crise, e isso tem se tornado uma realidade durante esse período de pandemia (BOUTOLEAU-BRETONNIERE C, et al., 2020). Diante do surgimento do coronavírus 2019 (COVID-19), esses idosos já debilitados, se tornaram ainda mais vulneráveis. Dessa forma, a associação entre idade avançada, Alzheimer e COVID-19 desperta inúmeras preocupações (WU Z e MCGOONGAN JM, 2020).

Além do risco de exposição, as manifestações da DA, principalmente a perda de memória, tornam difíceis para os idosos se protegerem adequadamente, visto que eles podem esquecer das medidas de proteção necessárias, sem falar do impacto do isolamento social desses idosos na saúde no geral e na sua família (WANG H, et al., 2020).

Dessa forma, estudar os impactos que o COVID-19 pode causar nos pacientes com DA é de extrema importância, para prevenir futuros agravamentos na saúde de idosos já vulneráveis e ser melhor investigado. Com isso, o objetivo desse estudo foi analisar através de uma revisão narrativa o impacto do isolamento social diante da pandemia COVID-19 para o cliente idoso com doença de Alzheimer e sua família.

\section{REVISÃO BIBLIOGRÁFICA}

Segundo Vallamkondu J, et al. (2020) o impacto de ambas pandemias, SARS-CoV-2, e doença de Alzheimer, é uma grande preocupação, especialmente na China e nos Estados Unidos. Os efeitos da doença de Alzheimer, particularmente 0 da perda de memória, tornam difícil para os idosos se protegerem adequadamente do vírus, pois eles podem se esquecer de seguir as precauções necessárias.

Pessoas que vivem com DA têm acesso limitado a informações e fatos precisos sobre a pandemia COVID19. Eles podem ter dificuldade em lembrar os procedimentos de proteção. Ignorar os avisos e não ter medidas de quarentena suficientes pode expô-los a uma chance maior de infecção (WANG H, et al., 2020). Embora o envelhecimento fisiológico do cérebro seja caracterizado por neuroinflamação, poda sináptica e perda neuronal subjacente a um declínio dependente da idade no desempenho sensorial, cognitivo e motor, a infecção por SARS-CoV-2 pode acelerar esse processo (VALLAMKONDU J, et al., 2020).

Segundo um estudo realizado na França, foi possível constatar que entre os 38 pacientes, 10 apresentaram sintomas neuropsiquiátricos no período de isolamento. A função cognitiva desses 10 pacientes, analisada através do Miniexame do Estado Mental (MEEM), foi ruim quando comparado com a dos pacientes que não apresentaram sintomas neuropsiquiátricos (BOUTOLEAU-BRETONNIERE C, et al., 2020).

Além disso, entre todas as mudanças, o aumento da vulnerabilidade dos pacientes com DA à morte é uma das mais marcantes. Até $80 \%$ dos que morrem de COVID estão em cuidados de longo prazo, traduzindo-se na possibilidade de que mais de um terço da mortalidade seja de pessoas com DA (LI J, et al., 2020). Conforme evidenciado por El Haj M, et al. (2020), os participantes do estudo relataram maior depressão ( $p=$ $0,005)$ e ansiedade $(p=0,004)$ durante do que antes da crise da COVID-19. Esses aumentos podem ser atribuídos ao isolamento dos moradores e/ou às mudanças drásticas em sua vida diária e cuidados que recebem. 
Os pacientes com DA são vulneráveis a desastres e crises, por causa de suas deficiências neurocognitivas e rica sintomatologia neuropsiquiátrica. Isso é especialmente verdadeiro durante a pandemia do COVID-19. Cerca de $80 \%$ dos pacientes com DA podem exibir pelo menos um sintoma neuropsiquiátrico durante o curso de sua doença, incluindo depressão, ansiedade, apatia, agitação e alucinações (FERINI-STRAMBI L e SALSONE M, 2020).

Pacientes idosos com DA apresentam risco aumentado de morbidade grave, admissão em unidades de terapia intensiva e morte quando infectados com COVID-19. Consequentemente, têm sido altamente recomendados para aderir ao distanciamento social, aumentando a sobrecarga do cuidador e dos familiares. Além disso, essas medidas podem levar esses pacientes a uma deterioração cognitiva mais rápida e ao agravamento dos sintomas comportamentais e psicológicos da DA (CUFFARO L, et al., 2020).

Segundo Tsugawa A, et al. (2020) a atual pandemia da doença coronavírus 2019 afetou substancialmente os pacientes com DA, seus cuidadores e familiares. No entanto, descobriu-se que nem todos os pacientes com DA tinham medo da infecção por COVID-19, por não estarem cientes dos eventos atuais. Nesse contexto, por não estarem cientes dos eventos atuais, pacientes com DA podem não compreender as mudanças em sua rotina e ficar agitados porque sua rotina regular é importante e por isso os familiares ficam mais estressados, ansiosos e sobrecarregados (ABATE G, et al., 2020; TOUSI B, 2020).

Por conseguinte, os pacientes com DA geralmente apresentam múltiplas comorbidades em comparação com outros, e essas aflições aumentam o risco de hospitalizações e mortalidade por infecções virais e bacterianas (MIYASHITA S, et al., 2020). Em tempos normais, os indivíduos com DA já estão entre as pessoas mais vulneráveis na sociedade, dependendo da família ou cuidadores para sua sobrevivência, sobrecarregando os mesmos. Além disso, esta pandemia exacerba ainda mais a vulnerabilidade dos idosos, devido à morbimortalidade da COVID-19 e aos efeitos indiretos da pandemia nos apoios sociais e no sistema de saúde do qual dependem (BROWN EE, et al., 2020).

Em virtude dos idosos com DA correrem um risco maior de mortalidade após a infecção por SARS-CoV2, é necessário isolar esses indivíduos e limitar seu contato com outras pessoas durante esta pandemia. Assim, o isolamento, embora necessário, pode infelizmente acarretar um risco aumentado de declínio cognitivo em indivíduos idosos, conforme evidenciado por inúmeros estudos (NAUGHTON S, et al., 2020).

Em uma pesquisa realizada na Irlanda, a mulher de um paciente com Alzheimer descreve o efeito do isolamento em sua vida, sendo eles o aumento da ansiedade, estresse e isolamento (LARA B, et al., 2020). Dessa forma, a DA pode ser impactante não apenas para as pessoas afetas, como também para seus cuidadores e familiares, que ficam sobrecarregados, com muitos afazeres, cuidados, preocupações e responsabilidades (ROCHFORD-BRENNAN H e KEOGH F, 2020). E embora a pandemia tenha se tornado um desafio para todas as famílias, para as famílias com pacientes com DA torna-se ainda mais desafiador, pois estão lidando com uma condição neurodegenerativa crônica que afeta a cognição (ABATE G, et al., 2020).

Após a leitura minuciosa e análise do conteúdo discursivo dos artigos foram levantadas as seguintes categorias temáticas: O impacto do isolamento social diante da pandemia COVID-19 para o idoso com doença de Alzheimer e sua família; Participação do(a) enfermeiro(a) no cuidado ao idoso com Alzheimer e sua família diante do impacto do isolamento social pela pandemia COVID-19.

\section{O impacto do isolamento social diante da pandemia COVID-19 para o idoso com doença de Alzheimer e sua família}

Atualmente, mais de 50 milhões de pessoas convivem com a DA no mundo inteiro. A cada três segundos um novo caso de DA é diagnosticado e nos próximos 20 anos esse valor deve dobrar (LLOYD-SHERLOCK $P$, et al., 2020). Em uma sociedade em envelhecimento, a DA surgiu como uma patologia pandêmica e ao ser associado a outra pandemia, a do SARS-CoV-2, torna-se uma temática de grande relevância e preocupação (WANG H, et al., 2020). 
Em alguns lugares como nos Estados Unidos, os idosos com DA geralmente ficam em casas de repouso que podem ser exclusivas ou em suas próprias casas. Já na China a maioria dos idosos vivem em lares compartilhados, no qual o idoso fica com seus filhos e netos, aumentando o risco de exposição ao vírus (VALLAMKONDU J, et al., 2020).

Em virtude da pandemia, os idosos com DA, já vulneráveis, ficaram ainda mais e além do risco de exposição ao vírus, os efeitos da DA, em destaque o da perda de memória, não permitem com que os idosos se protejam da maneira adequada, pois os mesmos podem esquecer das medidas de proteção necessárias, entre elas a utilização de máscaras. Dessa forma, ignorar as medidas e não ter uma quarentena eficaz pode expô-los a uma maior chance de contaminação (WANG H, et al., 2020).

Em concordância a isso, Perry G (2020) afirma que com a COVID-19, a vulnerabilidade dos pacientes com DA aumentou, principalmente com relação a mortalidade, visto que até $80 \%$ dos que morrem com coronavírus estão em cuidados de longo prazo, traduzindo-se na possibilidade de que mais de um terço da mortalidade seja de pessoas com DA, uma vez que aproximadamente metade daqueles em cuidados de longo prazo têm a doença. Um dos fatores pelo qual esses pacientes são suscetíveis é a questão da coabitação, além disso, normalmente um paciente com DA apresenta fatores de risco que se alinham aos do COVID-19, como a diabetes e doença cardíaca.

Wang $\mathrm{H}$, et al. (2020) ainda acrescenta afirmando que para reduzir a chance de contaminação entre idosos, as autoridades vêm restringindo a visita em lares de idosos e instituições de longa permanência, juntamente com as medidas de distanciamento social e como resultado os idosos perderam o contato com seus familiares, residentes, amigos e cuidadores e deixaram de realizar as atividades em grupo e exercícios físicos, com isso ficaram ainda mais isolados. Além disso, idosos com DA possuem pouco conhecimento das tecnologias, dependendo muito da ajuda de outras pessoas, o que pode ser muito solitário, deixando-os retraídos (US CENTERS FOR DISEASE CONTROL AND PREVENTION, 2020).

Em consonância a isso, um estudo com 38 participantes com um diagnóstico clínico de provável DA constatou que em média $30 \%$ dos pacientes com DA apresentaram alterações neuropsiquiátricas durante 0 confinamento. A função cognitiva avaliada através do Miniexame do Estado Mental foi pior nesses 10 participantes e a duração do confinamento se relacionou a gravidade dos sintomas. Evidenciando assim que o confinamento pode agravar a situação dos idosos com DA (BOUTOLEAU-BRETONNIERE C, et al., 2020).

Um estudo realizado na França investigou efeitos das medidas contra o COVID-19 na saúde mental de participantes com DA que vivem em casas de repouso e constatou que houve maior depressão e ansiedade durante a crise da COVID-19 do que antes dela. O aumento desses sintomas revela os efeitos atribuídos ao isolamento e as mudanças drásticas que foram feitas na vida diária desses pacientes (EL HAJ M, et al., 2020).

Outros autores trazem em sua pesquisa que entre os agravamentos dos sintomas neuropsiquiátricos desses pacientes em isolamento incluem agitação, apatia, atividade motora aberrante, ansiedade, estresse e depressão (LARA B, et al., 2020). Além disso, foi constatado que em pacientes com DA os sintomas de infecção por COVID-19 como febre, dispneia e tosse são menos frequentes, apresentando principalmente diarreia e sonolência (ISAIA G, et al., 2020).

Outros fatores devem ser considerados, como a capacidade dos pacientes com DA de não compreenderem as mudanças que estão ocorrendo em sua rotina, visto que uma rotina regular é de extrema importância para proporcionar maior conforto. Deve-se analisar a necessidade de discutir com esses pacientes sobre a pandemia, dependendo do estágio do comprometimento cognitivo, pois isso pode ocasionar aumento da ansiedade, do estresse deixá-los mais agitados (TOUSI B, 2020).

Wang $\mathrm{H}$, et al. (2020) acrescenta afirmando que a hipóxia causada pela COVID-19 ocasiona o delirium, fato esse que pode complicar o quadro da DA. De fato, é notório a convergência entre os estudos com relação ao impacto do confinamento e do distanciamento social no desempenho neurocognitivo dos pacientes com DA e não só isso, mas também o impacto da contaminação do vírus nesses pacientes que estão sujeitos a uma maior deterioração repentina. 
Dessa forma, outros aspectos também devem ser levados em consideração, visto que devido ao isolamento social todo o processo de diagnóstico ao tratamento foi comprometido, afetando também a qualidade de vida dessas pacientes, o que também influencia da deterioração do quadro clínico (LYKETSOS CG, et al., 2011).

Além de todos esses impactos decorrentes do isolamento, segundo dados de 627 pacientes internados no Hospital Agudos na província de Bréscia, norte da Itália, em comparação com indivíduos sem DA, os pacientes afetados por DA apresentaram uma mortalidade mais elevada em cerca de $40 \%$. Dessa forma, esses dados sugerem que a DA, especialmente nos estágios avançados da doença, pode representar um importante fator de risco para mortalidade em pacientes com COVID-19 (COVINO M, et al., 2020).

De acordo com especialistas, apoiar cuidadores e familiares de idosos com DA é extremamente necessário no mundo todo, pois os mesmos ficam muito sobrecarregados e estressados em virtude da alta demanda de cuidados, pois devido ao isolamento social os cuidados precisam ser redobrados, pois isolados, os idosos com DA precisam desenvolver outras atividades para que não fiquem nervosos e ansiosos e também para que o processo de neurodegeneração sejam lentificado e tudo isso fica sob responsabilidade dos familiares e cuidadores (WANG H, et al., 2020).

Além disso, em estudo realizado com mulher de um paciente com Alzheimer, foi relatado por ela que durante esse período de isolamento, os cuidados com seu esposo se tornaram dobrados, aumentando as responsabilidades dela e consequentemente aumentando os níveis de estresse e ansiedade (LARA B, et al., 2020).

\section{Participação do(a) enfermeiro(a) no cuidado ao idoso com Alzheimer e sua família diante do impacto do isolamento social pela pandemia COVID-19}

Diante da pandemia ser um acontecimento recente, verificou-se a insuficiência de estudos que abordassem sobre a participação do(a) enfermeiro(a) no cuidado ao idoso com Alzheimer e sua família durante o isolamento social, o que se constitui como uma limitação do presente estudo, visto que não foram encontrados estudos que discorram sobre essa temática (CUFFARO L, et al., 2020).

Entretanto, em consideração a complexa interação entre a COVID-19 e a DA, conforme recomendações internacionais, sugere-se fornecer maior suporte para esses pacientes com DA, entre eles a atuação da equipe multidisciplinar e a utilização das tecnologias, através da implementação dos serviços da telessaúde. Esses serviços podem ser extremamente úteis no monitoramento remoto, no cuidado, acompanhamento e também no suporte aos idosos com DA (CUFFARO L, et al., 2020).

Nesse contexto da telessaúde, surgiu a telenfermagem, que tem crescido no Brasil, principalmente depois da resolução do COFEN no 634/2020, que autoriza e normatiza a teleconsulta de enfermagem como estratégia de combate à pandemia provocada pelo novo coronavírus, através da realização de consultas, esclarecimentos, encaminhamentos e orientações por meio da utilização de recursos audiovisuais e dados que permitam o intercâmbio à distância entre o enfermeiro e o paciente (COFEN, 2020).

Nas teleconsultas é obrigatório a identificação do enfermeiro e da clínica de enfermagem, a autorização do paciente ou do seu representante legal por meio do termo de consentimento, autorizando a realização da consulta, o histórico do paciente, a observação clínica, o diagnóstico de enfermagem, o plano de cuidados e a avaliação de enfermagem e/ou encaminhamentos (COFEN, 2020).

Dessa forma, por meio das consultas de enfermagem é possível acompanhar esse paciente com DA e dar o suporte necessário para ele e sua família, sem que os mesmos precisem sair de casa. Além disso deve-se fornecer apoio psicossocial, para diminuir o estresse tanto do paciente quanto dos familiares. Algumas das medidas sugeridas para auxiliar esses idosos nesse período foram: realização de exercícios de relaxamento ou meditação através de mídia eletrônica e consultas online com equipe multidisciplinar, incluindo o enfermeiro (WANG H, et al., 2020).

Através da consulta de enfermagem, mesmo que seja à distância, é papel do profissional enfermeiro(a) identificar o cuidador principal, observar a dinâmica familiar e estruturas sociais e econômicas e diante disso, 
realizar ações adequadas. O(a) enfermeiro(a) necessita estar capacitado(a) para orientar a família e os cuidadores, capacitá-los com relação as técnicas adequadas, esclarecer dúvidas com relação a patologia, tratamento e prognóstico do paciente, assim como orientar sobre atividades que podem ser realizadas para diminuir o impacto do isolamento nesses idosos (JOHNSON M, et al., 2012).

Munindo-se de conhecimento cientifico, o profissional de enfermagem, dentro de suas atribuições, pode e deve estimular a função cognitiva de pacientes com DA, auxiliando na diminuição da ansiedade e agitação, garantindo e promovendo a segurança física, melhorando a comunicação e promovendo melhor qualidade de vida para o paciente por meio da realização de atividades de autocuidado, suprindo necessidades de socialização, solicitando atividades de repouso e produzindo um plano nutricional adequado (DOMINGUES M, et al., 2009).

No que concerne os cuidados de enfermagem aos cuidadores, que incluem os familiares, os profissionais de enfermagem devem realizar atividades de educação em saúde, para esclarecer as possíveis dúvidas e orientar quanto aos cuidados que devem ser realizados com o paciente. Além disso, deve-se prestar uma assistência voltada para proteger os familiares dos sintomas depressivos decorrentes dos desafios de lidar com a morte de um ente, auxiliando-os no desenvolvimento de mecanismos para enfrentar as dificuldades (ARAÚJO CLO, et al., 2012).

Entretanto, com a pandemia, deve-se buscar alternativas para realização dessa assistência, sendo a telessaúde a principal alternativa. Porém, o desafio está nas barreiras que os idosos enfrentam na adoção de uma nova tecnologia devido a desafios cognitivos, físicos e financeiros ou à falta de familiaridade. Apenas quatro em cada dez idosos possuem um celular. Dessa forma, se não houver um familiar, cuidador ou outra pessoa para auxiliar nesse processo, o idoso fica impossibilitado de utilizar os serviços da telessaúde e em grande maioria das vezes o familiar também é idoso, normalmente o parceiro ou parceira, e também não possui conhecimento das tecnologias (ABATE G, et al., 2020).

\section{CONSIDERAÇÕES FINAIS}

Analisou-se o impacto do coronavírus no paciente idoso com DA e sua família, constatando que discutir sobre essa temática é de extrema importância, pois em virtude da pandemia, os idosos com DA ficaram ainda mais vulneráveis e a família mais sobrecarregada. Além disso, esses idosos perderam o contato com familiares, amigos e cuidadores, deixando de realizar atividades em grupo e exercícios, ficando ainda mais isolados, apresentando maiores alterações. Dessa forma, sugere-se fornecer maior suporte para esses idosos, familiares e cuidadores, entre eles a atuação da equipe multidisciplinar e a utilização das tecnologias. Por fim, o presente estudo poderá fornecer subsídios às ações de planejamento para prevenir futuros agravamentos, promovendo a qualidade de vida e melhora na assistência aos pacientes idosos acometidos pela DA.

\section{REFERÊNCIAS}

1. ABATE G, et al. Impact of COVID-19 on Alzheimer's Disease Risk: Viewpoint for Research Action. Healthcare, 2020; 8(3): 286.

2. ARAÚJO CLO, et al. Perfil de cuidadores de idosos com doença de Alzheimer. Revista Kairós Gerontologia, 2012; 15(2): 119-137.

3. BOUTOLEAU-BRETONNIERE C, et al. The Effects of Confinement on Neuropsychiatric Symptoms in Alzheimer's Disease During the COVID-19 Crisis. Journal of Alzheimer's Disease, 2020; 76(1).

4. BRASIL. Resolução CNS no 466, de 12 de dezembro de 2012. Aprova diretrizes e normas regulamentadoras de pesquisas envolvendo seres humanos. Diário Oficial da União, Poder executivo, Brasília, DF, jun. 2013.

5. BROWN EE, et al. Anticipating and Mitigating the Impact of the COVID-19 Pandemic on Alzheimer's Disease and Related Dementias. Am J Geriatr Psychiatry, 2020; 28(7): 712-721.

6. CONSELHO FEDERAL DE ENFERMAGEM (COFEN). Resolução Cofen no 634/2020. Autoriza e normativa a teleconsulta de enfermagem. 2020. Disponível em: http://www.cofen.gov.br/resolucao-cofen-no-06342020_78344.html\#: :text=Autoriza\%20e\%20normatiza\%2C\%20\%E2\%80\%9Cad\%20referendum,tecnol\%C3\%B3gic os\%2C\%20e\%20d\%C3\%A1\%20outras\%20provid\%C3\%AAncias. Acesso em: 5 mai 2021. 
7. COVINO M, et al. Clinical characteristics and prognostic factors in COVID-19 patients aged $\geq 80$ years. Geriatr Gerontol Int., 2020; 20(7): 704-708.

8. CUFFARO L, et al. Dementia care and COVID-19 pandemic: a necessary digital revolution. Neurol Sci., 2020; 17: 13.

9. DOMINGUES M, et al. Doença de Alzheimer: o perfil dos cuidadores que utilizam o serviço de apoio telefônico da ABRAz - Associação Brasileira de Alzheimer. O Mundo da Saúde, 2009; 33(1): 161-169.

10. EL HAJ M, et al. High depression and anxiety in people with Alzheimer's disease living in retirement homes during the covid-19 crisis. Psychiatry Res., 2020; 291: 113294.

11. FERINI-STRAMBI L, SALSONE M. COVID-19 and neurological disorders: are neurodegenerative or neuroimmunological diseases more vulnerable?. J Neurol., 2020; 21: 1-11.

12. HASCUP ER, HASCUP KN. Does SARS-CoV-2 infection cause chronic neurological complications?. GeroScience, 2020; 42(4): 1083-1087.

13. ISAIA G, et al. Atypical presentation of COVID-19 in an older adult with severe Alzheimer disease. Am J Giatr Psychiatry, 2020; 28(7): 790-791.

14. JOHNSON M, et al. Ligações NANDA NOC-NIC: Condições clínicas suporte ao raciocínio e assistência de qualidade. 3 ed. Rio de Janeiro: Elsevier, 2012; 448p.

15. LARA B, et al. Neuropsychiatric symptoms and quality of life in spanish patients with Alzheimer's disease during the COVID-19 lockdown. Eur J Neurol, 2020; 27(9): 1744-1747.

16. LI J et al. Resilience of Alzheimer's Disease to COVID-19. J Alzheimers Dis., 2020; 77(1): 67-73.

17. LLOYD-SHERLOCK $P$, et al. Bearing the brunt of covid-19: older people in low and middle income countries. BMJ (Clinical research ed.), 2020; 368: 1-2.

18. LYKETSOS CG, et al. Neuropsychiatric disturbance in Alzheimer's disease clusters into three groups: the cache county study. Int J Geriatr Psychiatry, 2001; 16(11): 1043-53.

19. MIYASHITA S, et al. Impact of dementia on clinical outcomes in elderly patients with coronavirus 2019 (COVID-19): an experience in New York. Geriatr Gerontol Int., 2020; 20(7): 732-734.

20. NAUGHTON S, et al. Potential Novel Role of COVID-19 in Alzheimer's Disease and Preventative Mitigation Strategies. Journal of Alzheimer's Disease, 2020; 76(1): 21-25.

21. PERRY G. Alzheimer's Disease Patients in the Crosshairs of COVID-19. Journal of Alzheimer's Disease, 2020; 76(1): $1-1$.

22. PRINCE M, et al. Recent global trends in the prevalence and incidence of dementia, and survival with dementia. Alzheimer's Res. Ther., 2016; 8(1): 23.

23. ROCHFORD-BRENNAN H, KEOGH F. Giving voice to those directly affected by the COVID-19 pandemic - the experience and reflections of a person with dementia. HRB Open Res., 2020; 3: 29.

24. ROTHER, Edna T. Revisão sistemática X revisão narrativa. Acta paul. enferm., 2007; 20(2): 1-2.

25. SÍRIO-LIBANÊS. Alzheimer atinge 1,2 milhão de brasileiros. 2018. Disponível em: https://www.hospitalsiriolibanes.org.br/imprensa/noticias/Paginas/Alzheimer-atinge-1,2-milh\%C3\%A3o-debrasileiros.aspx. Acesso em: 22 jul. 2021.

26. TOUSI B. Dementia Care in the Time of COVID-19 Pandemic - Cuidados com a demência na época da pandemia de COVID-19. Journal of Alzheimer's Disease, 2020; 76(2): 475-479.

27. TSUGAWA A, et al. Awareness of the COVID-19 Outbreak and Resultant Depressive Tendencies in Patients with Severe Alzheimer's Disease. Journal of Alzheimer's Disease, 2020; 77(2): 539-541.

28. US CENTERS FOR DISEASE CONTROL AND PREVENTION. CDC's recommendations for the next 30 days of mitigation strategies for Seattle-King, Pierce, and Snohomish Counties based on current situation with widespread COVID-19 transmission and affected health care facilities. 2020. Disponível em: https://www.cdc.gov/coronavirus/2019-ncov/downloads/Seattle_Community_Mitigation.pdf. Acesso em: 20 dez. 2020.

29. VALLAMKONDU J, et al. SARS-CoV-2 pathophysiology and assessment of coronaviruses in CNS diseases with a focus on therapeutic targets. Biochim Biophys Acta Mol Basis Dis., 2020; 1866(10): 165889.

30. WANG H, et al. Dementia care during COVID-19. Lancet, 2020; 395(10231): 1190-1191.

31. WU Z, MCGOOGAN JM. Characteristics of and importante lessons from the Coronavirus Disease 2019 (COVID-19) outbreak in China. JAMA, 2020; 323(13): 1239-1242. 